\title{
STRUCTURE CONSTANTS OF THE WEYL CALCULUS
}

\author{
WEN DENG
}

\begin{abstract}
We find some explicit bounds on the $\mathscr{L}\left(L^{2}\right)$-norm of pseudo-differential operators with symbols defined by a metric on the phase space. In particular, we prove that this norm depends only on the "structure constants" of the metric and a fixed semi-norm of the symbol. Analogous statements are made for the Fefferman-Phong inequality.
\end{abstract}

\section{Introduction}

The class of symbols $S_{1,0}^{m}$ consists of smooth functions $a$ defined on the phase space $\mathrm{R}^{n} \times \mathrm{R}^{n}$ such that for all multi-indices $\alpha, \beta$,

$$
\left|\left(\partial_{\xi}^{\alpha} \partial_{x}^{\beta} a\right)(x, \xi)\right| \leq C_{\alpha, \beta}(1+|\xi|)^{m-|\alpha|} .
$$

The best constants $C_{\alpha, \beta}$ in (1.1) are called the semi-norms of the symbol $a$ in the Fréchet space $S_{1,0}^{m}$. We have

Property 1.1. If $a$ is in $S_{1,0}^{0}$, then $a(x, D)$ defines a bounded operator on $L^{2}\left(\mathrm{R}^{n}\right)$.

One might ask some very natural questions: the operator norm $\|a(x, D)\|_{\mathscr{L}\left(L^{2}\left(\mathrm{R}^{n}\right)\right)}$ is bounded by which constant? Is it a semi-norm of the symbol $a$ ? If yes, then which semi-norm? Questions of the same type might be asked for the constant $C$ in the following inequality:

Property 1.2 (Fefferman-Phong inequality). If $a$ is a non-negative symbol belonging to $S_{1,0}^{2}$, then there exists $C>0$ such that, for all $u \in \mathscr{S}\left(\mathrm{R}^{n}\right)$,

$$
\operatorname{Re}\langle a(x, D) u, u\rangle_{L^{2}\left(\mathrm{R}^{n}\right)}+C\|u\|_{L^{2}\left(\mathrm{R}^{n}\right)}^{2} \geq 0 .
$$

We can pose similar questions in many other examples of classes of symbols, such as the semi-classical symbols, Shubin's class, etc. As a particular example,

Received 2 December 2010, in final form 14 September 2011. 
the class $\Sigma^{m}$, defined as the set of smooth functions $a$ on $\mathrm{R}^{n} \times \mathrm{R}^{n} \times \mathrm{R}^{+}$such that for all multi-indices $\alpha, \beta$,

$$
\forall x, \xi \in \mathrm{R}^{n}, \tau \in \mathrm{R}^{+}, \quad\left|\left(\partial_{\xi}^{\alpha} \partial_{x}^{\beta} a\right)(x, \xi, \tau)\right| \leq C_{\alpha, \beta}(1+|\xi|+\tau)^{m-|\alpha|},
$$

is useful for Carleman estimates. One would like to check the Property 1.1 and Property 1.2 independent of the parameter $\tau$.

Several authors like Bony [1], Boulkhemair [3], Lerner-Morimoto [7], have already considered these questions and they were able to identify the constants. The constants in Properties 1.1, 1.2 are always a constant $C_{n}$ times a semi-norm of the symbol, whose order depends only on the dimension $n$. Although the problem is well-understood for a single class of pseudo-differential calculus, including the class $S(m, g)$ developed by Hörmander, we want to address a more general and useful question, having in mind the class $\Sigma^{m}$ depending on the non-compact parameter $\tau \geq 0$ which is defined in (1.3) and is useful for Carleman estimates.

In this paper, we consider the Weyl quantization for pseudo-differential operators and we choose the framework with a metric $g$ on the phase space. The metric $g$ is assumed to be admissible, that is slowly varying, satisfying the uncertainty principle and is temperate (see Definition 2.1, 2.6 below). The so-called structure constants of $g$ are closely related to these properties. We can define very general classes of symbols $S(m, g)$ attached to the metric $g$ and a $g$-admissible weight $m$ (see Definition 2.3) and we have an effective symbolic calculus. The following results are classical: (see [5, chapter 18], [6, chapter 2])

$$
\begin{aligned}
L^{2} \text {-boundedness: } & a \in S(1, g) \Longrightarrow\left\|a^{w}\right\| \mathscr{L}\left(L^{2}\left(\mathrm{R}^{n}\right)\right) \leq C, \\
\text { Fefferman-Phong: } & a \in S\left(\lambda_{g}^{2}, g\right), a \geq 0 \Longrightarrow a^{w}+C \geq 0 .
\end{aligned}
$$

The question that we would like to address is the following: what happens if we change the metric $g$ but keep the same structure constants?

We intend to show that the constants involved in (1.4), (1.5) depend only on the structure constants of the metric $g$ and a fixed semi-norm of $a$. Since it may happen that the metric $g$ depends on a non-compact parameter with uniform structure constants (e.g. the class $\Sigma^{m}$ ), this fact is useful explicitly or implicitly in many examples where these metrics are used and it seems useful to rely on a more stable argument than referring to "inspection of the proofs".

REMARK 1.3. An abstract functional analysis argument does not seem to work. Our method is to follow the proofs, by carefully computing all the constants. 


\section{Metric on the phase space}

In this section, we introduce the definitions of the admissible metric and exhibit its properties. We use the Weyl quantization which associates to a symbol $a$ the operator $a^{w}$ defined by

$$
\left(a^{w} u\right)(x)=\iint e^{2 i \pi(x-y) \cdot \xi} a\left(\frac{x+y}{2}, \xi\right) u(y) d y d \xi .
$$

Consider the symplectic space $\mathrm{R}^{2 n}$ equipped with the symplectic form $\sigma=$ $\sum_{j=1}^{n} d \xi^{j} \wedge d x^{j}$. Given a positive-definite quadratic form $\Gamma$ on $\mathrm{R}^{2 n}$, we define

$$
\Gamma^{\sigma}(T)=\sup _{\Gamma(Y)=1} \sigma(T, Y)^{2},
$$

which is also a positive-definite quadratic form. Let $g$ be a measurable map from $\mathrm{R}^{2 n}$ into the cone of positive-definite quadratic forms on $\mathrm{R}^{2 n}$, i.e., for each $X \in \mathrm{R}^{2 n}, g_{X}$ is a positive definite quadratic form on $\mathrm{R}^{2 n}$.

Definition 2.1 (Slowly varying metric). We say that $g$ is a slowly varying metric on $\mathrm{R}^{2 n}$, if there exists $C_{0} \geq 1$ such that for all $X, Y, T \in \mathrm{R}^{2 n}$,

$$
g_{X}(X-Y) \leq C_{0}^{-1} \Longrightarrow C_{0}^{-1} \leq \frac{g_{X}(T)}{g_{Y}(T)} \leq C_{0} .
$$

Definition 2.2 (Slowly varying weight). Let $g$ be a slowly varying metric on $\mathrm{R}^{2 n}$. A function $m: \mathrm{R}^{2 n} \rightarrow(0,+\infty)$ is called a $g$-slowly varying weight if there exists $\mu_{m} \geq 1$ such that for all $X, Y \in \mathrm{R}^{2 n}$,

$$
g_{X}(Y-X) \leq \mu_{m}^{-1} \Longrightarrow \mu_{m}^{-1} \leq \frac{m(X)}{m(Y)} \leq \mu_{m} .
$$

Definition 2.3 (Class of symbols). Let $g$ be a slowly varying metric on $\mathrm{R}^{2 n}$ and $m$ be a $g$-slowly varying weight. The class of symbols $S(m, g)$ is defined as the subset of functions $a \in C^{\infty}\left(\mathrm{R}^{2 n}\right)$ satisfying that for all $k \in \mathrm{N}$, there exists $C_{k}>0$ such that for all $X, T_{1}, \ldots, T_{k} \in \mathrm{R}^{2 n}$,

$$
\left|a^{(k)}(X)\left(T_{1}, \ldots, T_{k}\right)\right| \leq C_{k} m(X) \prod_{1 \leq j \leq k} g_{X}\left(T_{j}\right)^{1 / 2} .
$$

For $a \in S(m, g), l \in \mathrm{N}$, we denote

$$
\|a\|_{S(m, g)}^{(l)}=\max _{0 \leq k \leq l} \sup _{X, T_{j} \in \mathrm{R}^{2 n}, g_{X}\left(T_{j}\right)=1}\left|a^{(k)}(X)\left(T_{1}, \ldots, T_{k}\right)\right| m(X)^{-1} .
$$


The space $S(m, g)$ equipped with the countable family of semi-norms $\left(\|\cdot\|_{S(m, g)}^{(l)}\right)_{l \in \mathrm{N}}$ is a Fréchet space.

For a slowly varying metric $g$ on the phase space $\mathrm{R}^{2 n}$, we can introduce some partition of unity related to $g$. Define the $g$-ball near $X \in \mathrm{R}^{2 n}$

$$
U_{X, r}=\left\{Y, g_{X}(X-Y) \leq r^{2}\right\},
$$

we have the following theorem, which is Theorem 2.2.7 in [6].

THEOREM 2.4 (Partition of unity). Let $g$ be a slowly varying metric on $\mathrm{R}^{2 n}$ and $C_{0}>0$ given in (2.3). Then for all $r \in\left(0, C_{0}^{-1 / 2}\right]$, there exists a family $\left(\varphi_{Y}\right)_{Y \in \mathrm{R}^{2 n}}$ of smooth functions supported in $U_{Y, r}$ such that

$$
\begin{gathered}
\forall k \in \mathrm{N}, \quad \sup _{Y \in \mathrm{R}^{2 n}}\left\|\varphi_{Y}\right\|_{S(1, g)}^{(k)} \leq C\left(k, r, n, C_{0}\right), \\
\forall X \in \mathrm{R}^{2 n}, \quad \int_{\mathrm{R}^{2 n}} \varphi_{Y}(X)\left|g_{Y}\right|^{1 / 2} d Y=1,
\end{gathered}
$$

where $C\left(k, r, n, C_{0}\right)$ is a positive constant depending only on $k, r, n, C_{0}$ and $\left|g_{Y}\right|$ is the determinant of $g_{Y}$ with respect to the standard Euclidean norm.

Proof. As in the proof of Theorem 2.2.7 in [6], let $\chi_{0} \in C_{0}^{\infty}\left(\mathrm{R}_{+} ;[0,1]\right)$ non-increasing such that $\chi_{0}(t)=1$ on $t \leq 1 / 2, \chi_{0}(t)=0$ on $t \geq 1$. Define for $r \in\left(0, C_{0}^{-1 / 2}\right]$,

$$
\omega(X, r)=\int_{\mathrm{R}^{2 n}} \underbrace{\chi_{0}\left(r^{-2} g_{Y}(X-Y)\right)}_{=\omega_{Y}(X)}\left|g_{Y}\right|^{1 / 2} d Y .
$$

Since $\omega_{Y}(X)$ is supported in $U_{Y, r}$ and $\chi_{0}$ is non-increasing, by (2.3) we have

$$
\begin{aligned}
\omega(X, r) & \geq \int_{R^{2 n}} \chi_{0}\left(r^{-2} C_{0} g_{X}(X-Y)\right) C_{0}^{-n}\left|g_{X}\right|^{1 / 2} d Y \\
& =\int_{\mathrm{R}^{2 n}} \chi_{0}\left(|Z|^{2}\right) d Z C_{0}^{-2 n} r^{2 n},
\end{aligned}
$$

and an estimate from above of the same type, i.e., there exists a positive constant $C_{1}=C_{1}\left(r, n, C_{0}\right)$ such that

$$
C_{1}^{-1} \leq \omega(X, r) \leq C_{1} .
$$

Now let us check the derivatives of $\omega_{Y}(X)$. Using the notation $\langle,\rangle_{Y}$ the innerproduct associated to $g_{Y}$, we have

$$
\omega_{Y}^{\prime}(X) T=\chi_{0}^{\prime}\left(r^{-2} g_{Y}(X-Y)\right) r^{-2}\langle X-Y, T\rangle_{Y},
$$


and by induction, for $k \geq 1, T \in \mathrm{R}^{2 n}, \omega_{Y}^{(k)}(X) T^{k}$ is a finite sum of terms of type

$$
c_{p, k} \chi_{0}^{(p)}\left(r^{-2} g_{Y}(X-Y)\right) r^{-2 p}\langle X-Y, T\rangle_{Y}^{2 p-k} g_{Y}(T)^{k-p},
$$

where $c_{p, k}$ is a constant depending only on $p, k$ and $p \in[k / 2, k] \cap \mathrm{N}$. Since the support of $\chi_{0}^{(p)}$ is included in $[0,1]$ and $r^{2} \leq C_{0}^{-1}$, the term (2.9) can be bounded from above by

$$
c_{p, k}\left\|\chi_{0}^{(p)}\right\|_{L^{\infty}} r^{-2 p}\left(r^{2}\right)^{(2 p-k) / 2} C_{0}^{k / 2} g_{X}(T)^{k / 2},
$$

so that for all $k \geq 1,\left|\omega_{Y}^{(k)}(X) T^{k}\right| \leq C\left(k, r, C_{0}\right) g_{X}(T)^{k / 2}$. This implies that $\omega_{Y}$ is in $S(1, g)$ and moreover,

$$
\forall k \in \mathrm{N}, \quad \sup _{Y \in \mathrm{R}^{2 n}}\left\|\omega_{Y}\right\|_{S(1, g)}^{(k)} \leq C\left(k, r, C_{0}\right) .
$$

Now we choose a non-negative function $\chi_{1} \in C_{0}^{\infty}\left(\mathrm{R}_{+} ;[0,1]\right)$ such that $\chi_{1}(t)=$ 1 on $t \leq 1$, then

$$
\begin{aligned}
\left|\omega^{(k)}(X, r) T^{k}\right| & =\left.\left|\int_{\mathrm{R}^{2 n}} \omega_{Y}^{(k)}(X) T^{k} \chi_{1}\left(r^{-2} g_{Y}(X-Y)\right)\right| g_{Y}\right|^{1 / 2} d Y \mid \\
& \leq \sup _{Y \in \mathrm{R}^{2 n}}\left\|\omega_{Y}\right\|_{S(1, g)}^{(k)} g_{X}(T)^{k / 2} \int_{\mathrm{R}^{2 n}} \chi_{1}\left(r^{-2} g_{Y}(X-Y)\right)\left|g_{Y}\right|^{1 / 2} d Y \\
& \leq C\left(k, r, n, C_{0}\right) g_{X}(T)^{k / 2},
\end{aligned}
$$

which implies that $\omega(\cdot, r)$ is a symbol in $S(1, g)$ with $\|\omega(\cdot, r)\|_{S(1, g)}^{(k)} \leq$ $C^{\prime}\left(k, r, n, C_{0}\right)$. Since $\omega$ is bounded from below by $C_{1}^{-1}$, the function $\omega(\cdot, r)^{-1}$ is also in $S(1, g)$ and

$$
\left\|\omega(\cdot, r)^{-1}\right\|_{S(1, g)}^{(k)} \leq C^{\prime \prime}\left(k, r, n, C_{0}\right) .
$$

We define

$$
\varphi_{Y}(X)=\omega_{Y}(X) \omega(X, r)^{-1},
$$

then the estimate (2.7) follows from (2.10), (2.11) and moreover, the family $\left(\varphi_{Y}\right)_{Y \in \mathrm{R}^{2 n}}$ satisfies the requirements of Theorem 2.4.

A direct consequence of Theorem 2.4 is the following.

PROPOSITION 2.5. Let $g$ be a slowly varying metric on $\mathrm{R}^{2 n}$ and $m$ be a $g$ slowly varying weight. Let $C_{0}, \mu_{m}$ be given in (2.3), (2.4) respectively. Let a be a symbol in $S(m, g)$. Then for all $0<r \leq \min \left(C_{0}^{-1 / 2}, \mu_{m}^{-1 / 2}\right)$,

$$
a(X)=\int_{\mathrm{R}^{2 n}} a_{Y}(X)\left|g_{Y}\right|^{1 / 2} d Y
$$


where $a_{Y}$ has support included in $U_{Y, r}$ and

$$
\forall k \in \mathrm{N}, \quad \sup _{Y \in \mathrm{R}^{2 n}}\left\|a_{Y}\right\|_{S\left(m(Y), g_{Y}\right)}^{(k)} \leq C\left(k, r, C_{0}, n, \mu_{m}\right)\|a\|_{S(m, g)}^{(k)} .
$$

Proof. Define $a_{Y}(X)=a(X) \varphi_{Y}(X)$. Since $\varphi_{Y}$ is supported in $U_{Y, r}$, we have, for $k \geq 0, X \in U_{Y, r}, T \in \mathrm{R}^{2 n}$,

$$
\begin{aligned}
\left|a_{Y}^{(k)}(X) T^{k}\right| & =\left|\sum_{0 \leq l \leq k}\left(\begin{array}{c}
k \\
l
\end{array}\right) a^{(l)}(X) T^{l} \cdot \varphi_{Y}^{(k-l)}(X) T^{k-l}\right| \\
& \leq \sum_{0 \leq l \leq k} c_{k, l}\|a\|_{S(m, g)}^{(l)} m(X) g_{X}(T)^{l / 2}\left\|\varphi_{Y}\right\|_{S(1, g)}^{(k-l)} g_{X}(T)^{(k-l) / 2} \\
& \leq C(k)\|a\|_{S(m, g)}^{(k)}\left\|\varphi_{Y}\right\|_{S(1, g)}^{(k)} m(X) g_{X}(T)^{k / 2} \\
& \leq C(k) \mu_{m} C_{0}^{k / 2}\|a\|_{S(m, g)}^{(k)}\left\|\varphi_{Y}\right\|_{S(1, g)}^{(k)} m(Y) g_{Y}(T)^{k / 2}
\end{aligned}
$$

which completes the proof.

For two positive-definite quadratic forms $\Gamma_{1}, \Gamma_{2}$ on $\mathrm{R}^{2 n}$, the harmonic mean $\Gamma_{1} \wedge \Gamma_{2}$ is defined by

$$
\Gamma_{1} \wedge \Gamma_{2}=2\left(\Gamma_{1}^{-1}+\Gamma_{2}^{-1}\right)^{-1},
$$

which is also a positive-definite quadratic form on $\mathrm{R}^{2 n}$.

Definition 2.6 (Admissible metric). We say that $g$ is an admissible metric on $\mathrm{R}^{2 n}$ if $g$ is slowly varying (see Definition 2.1) and there exist $C_{0}^{\prime}>0$, $N_{0} \in \mathrm{N}$ such that for all $X, Y, T \in \mathrm{R}^{2 n}$,

(2.14) uncertainty principle $g_{X}(T) \leq g_{X}^{\sigma}(T)$,

(2.15) temperance $g_{X}(T) \leq C_{0}^{\prime} g_{Y}(T)\left(1+\left(g_{X}^{\sigma} \wedge g_{Y}^{\sigma}\right)(X-Y)\right)^{N_{0}}$,

where $g^{\sigma}$ is given by (2.2) and $\wedge$ given by (2.13).

We may suppose $C_{0}^{\prime}=C_{0}$ in the sequel, where $C_{0}$ is given in (2.3). Then the constants $\left(C_{0}, N_{0}\right)$ appearing in (2.3), (2.15) are called the structure constants of the metric $g$.

Definition 2.7 (Admissible weight). Suppose that $g$ is an admissible metric on $\mathrm{R}^{2 n}$. A function $m: \mathrm{R}^{2 n} \rightarrow(0,+\infty)$ is called a $g$-admissible weight if $m$ is a $g$-slowly varying weight (see Definition 2.2) and there exist $\mu_{m}>0$, $v_{m} \in \mathrm{N}$ such that for all $X, Y \in \mathrm{R}^{2 n}$,

$$
m(X) \leq \mu_{m} m(Y)\left(1+\left(g_{X}^{\sigma} \wedge g_{Y}^{\sigma}\right)(X-Y)\right)^{\nu_{m}} .
$$


The constants $\left(\mu_{m}, v_{m}\right)$ appearing in (2.4), (2.16) are called the structure constants of the $g$-admissible weight $m$.

Let $g$ be an admissible metric on $\mathrm{R}^{2 n}$. We define for $X \in \mathrm{R}^{2 n}$,

$$
\lambda_{g}(X)=\inf _{T \neq 0}\left(\frac{g_{X}^{\sigma}(T)}{g_{X}(T)}\right)^{1 / 2} .
$$

Then the uncertainty principle (2.14) can be expressed by

$$
g_{X} \leq \lambda_{g}(X)^{-2} g_{X}^{\sigma}, \quad \lambda_{g}(X) \geq 1 .
$$

Lemma 2.8 ([6, Remark 2.2.17]). For any $s \in \mathrm{R}, \lambda_{g}^{s}$ is an admissible weight, with structure constants $\left(\mu_{\lambda_{g}^{s}}, \nu_{\lambda_{g}^{s}}\right)$ in (2.4), (2.16) depending only on the structure constants of the metric $g\left(C_{0}, N_{0}\right)$.

Proof. We first verify that $\lambda_{g}^{s}$ is a $g$-slowly varying weight. For $g_{X}(X-$ $Y) \leq C_{0}^{-1}, T \in \mathrm{R}^{2 n}$, we have

$$
C_{0}^{-1} g_{X}(T) \leq g_{Y}(T) \leq C_{0} g_{X}(T), \quad C_{0}^{-1} g_{X}^{\sigma}(T) \leq g_{Y}^{\sigma}(T) \leq C_{0} g_{X}^{\sigma}(T),
$$

which implies

$$
C_{0}^{-2} \frac{g_{X}^{\sigma}(T)}{g_{X}(T)} \leq \frac{g_{Y}^{\sigma}(T)}{g_{Y}(T)} \leq C_{0}^{2} \frac{g_{X}^{\sigma}(T)}{g_{X}(T)} .
$$

Taking the infimum with respect to $T$, we get

$$
C_{0}^{-2} \lambda_{g}(X)^{2} \leq \lambda_{g}(Y)^{2} \leq C_{0}^{2} \lambda_{g}(X)^{2},
$$

so that $\lambda_{g}$ is $g$-slowly varying with $\mu_{\lambda_{g}}=C_{0}$ and so is $\lambda_{g}^{s}$ with $\mu_{\lambda_{g}^{s}}=C_{0}^{|s|}$. Next we check that $\lambda_{g}^{s}$ is temperate. We have for all $X, Y, T \in \mathrm{R}^{2 n}$,

$$
\begin{aligned}
& g_{X}(T) \geq C_{0}^{-1} g_{Y}(T)\left(1+\left(g_{X}^{\sigma} \wedge g_{Y}^{\sigma}\right)(X-Y)\right)^{-N_{0}}, \\
& g_{X}^{\sigma}(T) \leq C_{0} g_{Y}^{\sigma}(T)\left(1+\left(g_{X}^{\sigma} \wedge g_{Y}^{\sigma}\right)(X-Y)\right)^{N_{0}},
\end{aligned}
$$

which gives

$$
\lambda_{g}(X)^{2} \leq C_{0}^{2} \lambda_{g}(Y)^{2}\left(1+\left(g_{X}^{\sigma} \wedge g_{Y}^{\sigma}\right)(X-Y)\right)^{2 N_{0}} .
$$

Thus $\lambda_{g}$ is temperate with $\nu_{\lambda_{g}}=N_{0}$ and so is $\lambda_{g}^{s}$ with $\nu_{\lambda_{g}^{s}}=|s| N_{0}$. This completes the proof of Lemma 2.8.

The composition $a \sharp b$ of two symbols is defined by $a^{w} b^{w}=(a \sharp b)^{w}$ and we have, with the notations $[X, Y]=\sigma(X, Y), D=(2 i \pi)^{-1} \partial$,

$$
(a \sharp b)(X)=2^{2 n} \iint_{\mathrm{R}^{2 n} \times \mathrm{R}^{2 n}} a(Y) b(Z) e^{-4 i \pi[X-Y, X-Z]} d Y d Z,
$$




$$
(a \sharp b)(X)=\exp \left(i \pi\left[D_{Y}, D_{Z}\right]\right)(a(Y) b(Z))_{\mid Y=Z=X} .
$$

For $a \in S\left(m_{1}, g\right), b \in S\left(m_{2}, g\right)$, we have the asymptotic expansion

$$
a \sharp b(x, \xi)=\sum_{0 \leq k<p} w_{k}(a, b)(x, \xi)+r_{p}(a, b)(x, \xi),
$$

with

(2.21) $w_{k}(a, b)=2^{-k} \sum_{|\alpha|+|\beta|=k} \frac{(-1)^{|\beta|}}{\alpha ! \beta !} D_{\xi}^{\alpha} \partial_{x}^{\beta} a D_{\xi}^{\beta} \partial_{x}^{\alpha} b \in S\left(m_{1} m_{2} \lambda_{g}^{-k}, g\right)$,

$$
\begin{gathered}
r_{p}(a, b)(X)=R_{p}(a(X) \otimes b(Y))_{\mid Y=X} \in S\left(m_{1} m_{2} \lambda_{g}^{-p}, g\right) \\
R_{p}=\int_{0}^{1} \frac{(1-\theta)^{p-1}}{(p-1) !} \exp \frac{\theta}{4 i \pi}\left[\partial_{X}, \partial_{Y}\right] d \theta\left(\frac{1}{4 i \pi}\left[\partial_{X}, \partial_{Y}\right]\right)^{p} .
\end{gathered}
$$

Notice $w_{1}(a, b)=\frac{1}{4 i \pi}\{a, b\}$, where $\{$,$\} denotes the Poisson bracket, so that$ the asymptotic (2.20) at $p=2$ is

$$
a \sharp b=a b+\frac{1}{4 i \pi}\{a, b\}+r_{2}(a, b) .
$$

Definition 2.9 (The main distance function). Let $g$ be an admissible metric on $\mathrm{R}^{2 n}$. Define the main distance function, for $r>0, X, Y \in \mathrm{R}^{2 n}$,

$$
\delta_{r}(X, Y)=1+\left(g_{X}^{\sigma} \wedge g_{Y}^{\sigma}\right)\left(U_{X, r}-U_{Y, r}\right),
$$

where $U_{X, r}$ is given in (2.6) and

$$
g(U-V)=\inf _{X \in U, Y \in V} g(X-Y) .
$$

Lemma 2.10 ([6, Lemma 2.2.24], Integrability of $\delta_{r}$ ). Let $g$ be an admissible metric with structure constants $\left(C_{0}, N_{0}\right)$. Then there exist positive constants $N_{1}=N_{1}\left(n, C_{0}, N_{0}\right), C=C\left(n, C_{0}, N_{0}\right)$ such that for all $r \in\left(0, C_{0}^{-1 / 2}\right]$,

$$
\sup _{X \in \mathrm{R}^{2 n}} \int_{\mathrm{R}^{2 n}} \delta_{r}(X, Y)^{-N_{1}}\left|g_{Y}\right|^{1 / 2} d Y \leq C<+\infty .
$$


Proof. Suppose $r \leq C_{0}^{-1 / 2}$. Using the slowness and temperance of $g$, for $X^{\prime} \in U_{X, r}, Y^{\prime} \in U_{Y, r}, \bar{T} \in \mathrm{R}^{2 n}$, we have

$$
\begin{aligned}
\left(g_{X}^{\sigma} \wedge\right. & \left.g_{Y}^{\sigma}\right)(T) \geq C_{0}^{-1}\left(g_{X^{\prime}}^{\sigma} \wedge g_{Y^{\prime}}^{\sigma}\right)(T) \\
& \geq C_{0}^{-2} g_{X^{\prime}}^{\sigma}(T)\left(1+\left(g_{X^{\prime}}^{\sigma} \wedge g_{Y^{\prime}}^{\sigma}\right)\left(X^{\prime}-Y^{\prime}\right)\right)^{-N_{0}} \\
& \geq C_{0}^{-3} g_{X}^{\sigma}(T)\left(1+C_{0}\left(g_{X}^{\sigma} \wedge g_{Y}^{\sigma}\right)\left(X^{\prime}-Y^{\prime}\right)\right)^{-N_{0}} \\
& \geq C_{0}^{-3-N_{0}} g_{X}^{\sigma}(T)\left(1+\left(g_{X}^{\sigma} \wedge g_{Y}^{\sigma}\right)\left(X^{\prime}-Y^{\prime}\right)\right)^{-N_{0}}
\end{aligned}
$$

Taking the infimum in $X^{\prime} \in U_{X, r}, Y^{\prime} \in U_{Y, r}$, we get

$$
g_{X}^{\sigma}(T) \leq C_{0}^{3+N_{0}} \delta_{r}(X, Y)^{N_{0}}\left(g_{X}^{\sigma} \wedge g_{Y}^{\sigma}\right)(T)
$$

We have also

$$
\begin{aligned}
\frac{g_{X}(T)}{g_{Y}(T)} \leq C_{0}^{2} \frac{g_{X^{\prime}}(T)}{g_{Y^{\prime}}(T)} & \leq C_{0}^{3}\left(1+\left(g_{X^{\prime}}^{\sigma} \wedge g_{Y^{\prime}}^{\sigma}\right)\left(X^{\prime}-Y^{\prime}\right)\right)^{N_{0}} \\
& \leq C_{0}^{3}\left(1+C_{0}\left(g_{X}^{\sigma} \wedge g_{Y}^{\sigma}\right)\left(X^{\prime}-Y^{\prime}\right)\right)^{N_{0}} \\
& \leq C_{0}^{3+N_{0}}\left(1+\left(g_{X}^{\sigma} \wedge g_{Y}^{\sigma}\right)\left(X^{\prime}-Y^{\prime}\right)\right)^{N_{0}}
\end{aligned}
$$

By taking the infimum in $X^{\prime}, Y^{\prime}$, we get the following inequality

$$
\frac{g_{X}(T)}{g_{Y}(T)} \leq C_{0}^{3+N_{0}} \delta_{r}(X, Y)^{N_{0}} .
$$

Then

$$
\begin{aligned}
& 1+g_{X}(X-Y) \leq 1+3 g_{X}\left(X-X^{\prime}\right)+3 g_{X}\left(X^{\prime}-Y^{\prime}\right)+3 g_{X}\left(Y^{\prime}-Y\right) \\
& \quad \stackrel{(2.28)}{\leq} 3 C_{0}^{3+N_{0}} \delta_{r}(X, Y)^{N_{0}}\left(1+g_{X}\left(X-X^{\prime}\right)+g_{X}\left(X^{\prime}-Y^{\prime}\right)+g_{Y}\left(Y^{\prime}-Y\right)\right) \\
& \quad \leq 3 C_{0}^{3+N_{0}} \delta_{r}(X, Y)^{N_{0}}\left(1+2 r^{2}+g_{X}^{\sigma}\left(X^{\prime}-Y^{\prime}\right)\right) \\
& \quad \text { by (2.27) } 9 C_{0}^{6+2 N_{0}} \delta_{r}(X, Y)^{2 N_{0}}\left(1+\left(g_{X}^{\sigma} \wedge g_{Y}^{\sigma}\right)\left(X^{\prime}-Y^{\prime}\right)\right),
\end{aligned}
$$

so that $1+g_{X}(X-Y) \leq 9 C_{0}^{6+2 N_{0}} \delta_{r}(X, Y)^{2 N_{0}+1}$. In the other hand, we have

$$
\frac{\left|g_{Y}\right|^{1 / 2}}{\left|g_{X}\right|^{1 / 2}} \leq C_{0}^{n\left(3+N_{0}\right)} \delta_{r}(X, Y)^{n N_{0}}
$$


so that for $N_{1}=n N_{0}+(n+1)\left(2 N_{0}+1\right)>0$,

$$
\begin{aligned}
& \int_{\mathrm{R}^{2 n}} \delta_{r}(X, Y)^{-N_{1}}\left|g_{Y}\right|^{1 / 2} d Y \\
& \quad \leq C\left(n, C_{0}, N_{0}\right) \int_{\mathrm{R}^{2 n}} \delta_{r}(X, Y)^{-N_{1}+n N_{0}}\left|g_{X}\right|^{1 / 2} d Y \\
& \quad \leq C^{\prime}\left(n, C_{0}, N_{0}\right) \int_{\mathrm{R}^{2 n}}\left(1+g_{X}(X-Y)\right)^{-(n+1)}\left|g_{X}\right|^{1 / 2} d Y \\
& \quad=C^{\prime}\left(n, C_{0}, N_{0}\right) \int_{\mathrm{R}^{2 n}}\left(1+|Z|^{2}\right)^{-(n+1)} d Z<+\infty .
\end{aligned}
$$

The proof of the lemma is complete.

\section{3. $L^{2}$-boundedness}

In this section, we prove the $L^{2}$-boundedness of pseudo-differential operators with symbol in $S(1, g)$ and make precise the operator norms.

\subsection{The constant metric case}

PROPOSITION 3.1. Suppose that $g$ is a positive-definite quadratic form (constant metric) on $\mathrm{R}^{2 n}$ with $g \leq g^{\sigma}$. Then there exists a constant $C(n)>0$ depending only on the dimension $n$ such that for all $a \in S(1, g)$,

$$
\left\|a^{w}\right\|_{\mathscr{L}\left(L^{2}\left(\mathrm{R}^{n}\right)\right)} \leq C(n)\|a\|_{S(1, g)}^{(2 n+1)} .
$$

Proof. Since $g$ is a constant metric, according to Lemma 4.4.25 in [6], there exist symplectic coordinates $(x, \xi)$ such that

$$
g=\sum_{1 \leq j \leq n} \lambda_{j}^{-1}\left(\left|d x_{j}\right|^{2}+\left|d \xi_{j}\right|^{2}\right), \quad g^{\sigma}=\sum_{1 \leq j \leq n} \lambda_{j}\left(\left|d x_{j}\right|^{2}+\left|d \xi_{j}\right|^{2}\right),
$$

with $\lambda_{j}>0 . g \leq g^{\sigma}$ is expressed as

$$
\min _{1 \leq j \leq n} \lambda_{j} \geq 1
$$

As a result, we have $g \leq|d x|^{2}+|d \xi|^{2}:=\Gamma_{0}$, which implies $S(1, g) \subset$ $S\left(1, \Gamma_{0}\right)$ and for all $a \in S(1, g)$,

$$
\forall l \in \mathrm{N}, \quad\|a\|_{S\left(1, \Gamma_{0}\right)}^{(l)} \leq\|a\|_{S(1, g)}^{(l)} .
$$

By Theorem 1.1.4 in [6] and $a^{w}=\left(J^{1 / 2} a\right)(x, D)$, where $J^{t}$ is introduced in Lemma 4.1.2 in [6], we obtain that

$$
\left\|a^{w}\right\|_{\mathscr{L}\left(L^{2}\left(\mathrm{R}^{n}\right)\right)} \leq C(n)\|a\|_{S\left(1, \Gamma_{0}\right)}^{(2 n+1)},
$$


where $C(n)$ depends only on $n$. Together with (3.1), we complete the proof of the proposition.

\subsection{The general case}

THEOREM 3.2. Let $g$ be an admissible metric on $\mathrm{R}^{2 n}$ with structure constants $\left(C_{0}, N_{0}\right)$ (see Definition 2.6). Then there exist $C=C\left(n, C_{0}, N_{0}\right)>0$ and $l=l\left(n, C_{0}, N_{0}\right) \in \mathrm{N}$ such that for all $a \in S(1, g)$ (see Definition 2.3),

$$
\left\|a^{w}\right\| \mathscr{L}\left(L^{2}\left(\mathrm{R}^{n}\right)\right) \leq C\|a\|_{S(1, g)}^{(l)} .
$$

Proof. Using the partition in Proposition 2.5, we write

$$
a^{w}=\int_{\mathrm{R}^{2 n}} a_{Y}^{w}\left|g_{Y}\right|^{1 / 2} d Y,
$$

where $a_{Y}$ is supported in $U_{Y, r}$ and satisfies (2.12). By Proposition 3.1, we have $\sup _{Y}\left\|a_{Y}^{w}\right\|_{\mathscr{L}\left(L^{2}\left(\mathrm{R}^{n}\right)\right)} \leq C\left(r, n, C_{0}, N_{0}\right)\|a\|_{S(1, g)}^{(2 n+1)}<+\infty$. The following lemma is useful.

LeMmA 3.3 (Cotlar). Let $H$ be a Hilbert space and $(\Omega, \mathscr{A}, v)$ a measured space such that $v$ is a $\sigma$-finite positive measure. Let $\left(A_{y}\right)_{y \in \Omega}$ be a measurable family of bounded operators on $H$ such that

$$
\sup _{y \in \Omega} \int_{\Omega}\left\|A_{y}^{*} A_{z}\right\|_{\mathscr{L}(H)}^{1 / 2} d \nu(z) \leq M, \quad \sup _{y \in \Omega} \int_{\Omega}\left\|A_{y} A_{z}^{*}\right\|_{\mathscr{L}(H)}^{1 / 2} d \nu(z) \leq M .
$$

Then for all $u \in H$, we have

$$
\iint_{\Omega \times \Omega}\left|\left\langle A_{y} u, A_{z} u\right\rangle_{H}\right| d v(y) d v(z) \leq M^{2}\|u\|_{H}^{2},
$$

which implies the strong convergence of $A=\int_{\Omega} A_{y} d v(y)$ and $\|A\|_{\mathscr{L}(H)} \leq M$.

In order to apply Cotlar's lemma, we should estimate $\left\|\bar{a}_{Y}^{w} a_{Z}^{w}\right\|_{\mathscr{L}\left(L^{2}\left(\mathrm{R}^{n}\right)\right)}$, i.e., a semi-norm of $\bar{a}_{Y} \sharp a_{Z}$ in $S\left(1, g_{Y}+g_{Z}\right)$. Indeed, the following estimate holds.

LeMmA 3.4. Let $g, a_{Y}$ be as above. For any $k, N \in \mathrm{N}$, there exist $C=$ $C(k, N, n)>0, l=l(k, N, n) \in \mathrm{N}$ such that

$$
\left\|\bar{a}_{Y} \sharp a_{Z}\right\|_{S\left(1, g_{Y}+g_{Z}\right)}^{(k)} \leq C\left\|\bar{a}_{Y}\right\|_{S\left(1, g_{Y}\right)}^{(l)}\left\|a_{Z}\right\|_{S\left(1, g_{Z}\right)}^{(l)} \delta_{r}(Y, Z)^{-N} .
$$

We use some biconfinement estimates, which can be found in [6, section 2.3], to prove Lemma 3.4. 
Definition 3.5 (Confined symbols). Let $g$ be a positive-definite quadratic form on $\mathrm{R}^{2 n}$ such that $g \leq g^{\sigma}$. Let $a$ be a smooth function on $\mathrm{R}^{2 n}$ and $U \subset \mathrm{R}^{2 n}$. We say that $a$ is $g$-confined in $U$, if for all $k, N \in \mathrm{N}$, there exits $C_{k, N}>0$ such that for all $X, T \in \mathrm{R}^{2 n}$,

$$
\left|a^{(k)}(X) T^{k}\right| \leq C_{k, N} g(T)^{k / 2}\left(1+g^{\sigma}(X-U)\right)^{-N / 2} .
$$

We denote

$$
\|a\|_{g, U}^{(k, N)}=\sup _{X, T \in \mathrm{R}^{2 n}, g(T)=1}\left|a^{(k)}(X) T^{k}\right|\left(1+g^{\sigma}(X-U)\right)^{N / 2},
$$

and

$$
\|a\|_{g, U}^{(l)}=\max _{k \leq l}\|a\|_{g, U}^{(k, l)} .
$$

Theorem 3.6 ([6, Theorem 2.3.2], biconfinement estimate). Let $g_{1}, g_{2}$ be two positive-definite quadratic forms on $\mathrm{R}^{2 n}$ such that $g_{j} \leq g_{j}^{\sigma}$. Let $a_{j}, j=1,2$ be $g_{j}$-confined in $U_{j}$, a $g_{j}$-ball of radius $\leq 1$. Then for all $k, N \in \mathrm{N}$, for all $X, T \in \mathrm{R}^{2 n}$,

(3.5) $\left|\left(a_{1} \sharp a_{2}\right)^{(k)}(X) T^{k}\right|$

$\leq A_{k, N}\left(g_{1}+g_{2}\right)(T)^{k / 2}\left(1+\left(g_{1}^{\sigma} \wedge g_{2}^{\sigma}\right)\left(X-U_{1}\right)+\left(g_{1}^{\sigma} \wedge g_{2}^{\sigma}\right)\left(X-U_{2}\right)\right)^{-N / 2}$,

with $A_{k, N}=\gamma(k, N, n)\left\|a_{1}\right\|\left\|_{g_{1}, U_{1}}^{(l)}\right\| a_{2}\|\|_{g_{2}, U_{2}}^{(l)}, l=2 n+1+k+N$.

Now we begin the proof of Lemma 3.4.

Proof of Lemma 3.4. The symbol $a_{Y}$ is $g_{Y}$-confined in $U_{Y, r}$, since $a_{Y}$ is supported in the $g_{Y}$-ball $U_{Y, r}$. Moreover, we have

$$
\begin{gathered}
\forall k, N \in \mathrm{N}, \quad\left\|a_{Y}\right\|_{g_{Y}, U_{Y, r}}^{(k, N)}=\sup _{X \in U_{Y, r}, T \in \mathrm{R}^{2 n}, g_{Y}(T)=1}\left|a^{(k)}(X) T^{k}\right|, \\
\forall l \in \mathrm{N}, \quad\left\|a_{Y}\right\|_{g_{Y}, U_{Y, r}}^{(l)}=\max _{k \leq l}\left\|a_{Y}\right\|_{g_{Y}, U_{Y, r}}^{(k, l)}=\left\|a_{Y}\right\|_{S\left(1, g_{Y}\right)}^{(l)} .
\end{gathered}
$$

Applying (3.5) to $\bar{a}_{Y} \sharp a_{Z}$ and using the triangular inequality

$$
\left(g_{Y}^{\sigma} \wedge g_{Z}^{\sigma}\right)\left(X-U_{Y, r}\right)+\left(g_{Y}^{\sigma} \wedge g_{Z}^{\sigma}\right)\left(X-U_{Z, r}\right) \geq \frac{1}{2}\left(g_{Y}^{\sigma} \wedge g_{Z}^{\sigma}\right)\left(U_{Y, r}-U_{Z, r}\right),
$$

we get

$$
\begin{aligned}
\left|\left(\bar{a}_{Y} \sharp a_{Z}\right)^{(k)}(X) T^{k}\right| \leq \gamma(k, N, n)\left\|\bar{a}_{Y}\right\|_{S\left(1, g_{Y}\right)}^{(l)}\left\|a_{Z}\right\|_{S\left(1, g_{Z}\right)}^{(l)}\left(g_{Y}+g_{Z}\right)(T)^{k / 2} & \\
& \times\left(1+\frac{1}{2}\left(g_{Y}^{\sigma} \wedge g_{Z}^{\sigma}\right)\left(U_{Y, r}-U_{Z, r}\right)\right)^{-N / 2} .
\end{aligned}
$$


Using the definition of the distance $\delta_{r}$, we complete the proof of Lemma 3.4.

END OF THE PROOF OF THEOREM 3.2. Now by Proposition 3.1, Lemma 3.4 and the estimate (2.12), we obtain that for any $N>0$, there exists $l=$ $l(N, n) \in \mathrm{N}$ such that

$$
\begin{aligned}
\left\|\bar{a}_{Y}^{w} a_{Z}^{w}\right\|_{\mathscr{L}\left(L^{2}\left(\mathrm{R}^{n}\right)\right)} & \leq C(n)\left\|\bar{a}_{Y}^{w} a_{Z}^{w}\right\|_{S\left(1, g_{Y}+g_{Z}\right)}^{(2 n+1)} \\
& \leq C(N, n)\left\|\bar{a}_{Y}\right\|_{S\left(1, g_{Y}\right)}^{(l)}\left\|a_{Z}\right\|_{S\left(1, g_{Z}\right)}^{(l)} \delta_{r}(Y, Z)^{-N} \\
& \leq C\left(N, n, C_{0}\right)\left(\|a\|_{S(1, g)}^{(l)}\right)^{2} \delta_{r}(Y, Z)^{-N}
\end{aligned}
$$

The same inequality holds for $a_{Y} \sharp \bar{a}_{Z}$. Choose $N=2 N_{1}$, where $N_{1}$ is given in (2.26), so that

$$
\max \left\{\left\|\bar{a}_{Y}^{w} a_{Z}^{w}\right\|_{\mathscr{L}\left(L^{2}\left(\mathrm{R}^{n}\right)\right)}^{1 / 2},\left\|a_{Y}^{w} \bar{a}_{Z}^{w}\right\|_{\mathscr{L}\left(L^{2}\left(\mathrm{R}^{n}\right)\right)}^{1 / 2}\right\} \leq C\|a\|_{S(1, g)}^{(l)} \delta_{r}(Y, Z)^{-N_{1}},
$$

where $C=C\left(n, C_{0}, N_{1}\right)>0, l=l\left(n, N_{1}\right) \in \mathrm{N}$. Then together with Lemma 2.10, the assumptions of Cotlar's lemma are fulfilled with $M=$ $C\|a\|_{S(1, g)}^{(l)}$, and this completes the proof of Theorem 3.2.

\section{Fefferman-Phong inequality}

In this section, we prove that the constant in the Fefferman-Phong inequality depends only on the structure constants of the metric and a fixed semi-norm of the symbol.

THEOREM 4.1 (Fefferman-Phong inequality). Let $\mathrm{g}$ be an admissible metric on $\mathrm{R}^{2 n}$ with structure constants $\left(C_{0}, N_{0}\right)$ (see Definition 2.6). Let a be a nonnegative symbol in $S\left(\lambda_{g}^{2}, g\right)$ (see Definition 2.3 and (2.17)). Then the operator $a^{w}$ on $L^{2}\left(\mathrm{R}^{n}\right)$ is semi-bounded from below. More precisely, there exist $l=$ $l\left(n, C_{0}, N_{0}\right) \in \mathrm{N}, C=C\left(n, C_{0}, N_{0}\right)>0$ such that

$$
a^{w}+C\|a\|_{S\left(\lambda_{g}^{2}, g\right)}^{(l)} \geq 0 .
$$

\subsection{The constant metric case}

For the constant metric case, we use the results of Sjöstrand and refer the readers to [6, page 116] for the detailed proof.

Let $1=\sum_{j \in Z^{2 n}} \chi_{0}(X-j)$ be a partition of unity, $\chi_{0} \in C_{c}^{\infty}\left(\mathrm{R}^{2 n}\right)$. Denote $\chi_{j}(X)=\chi_{0}(X-j)$.

Proposition 4.2 ([6, Proposition 2.5.6]). Suppose a $\in \mathscr{S}\left(\mathrm{R}^{2 n}\right)$. We say that a belongs to the class $\mathscr{A}$ if $\omega_{a} \in L^{1}\left(\mathrm{R}^{2 n}\right)$, with $\omega_{a}(\Xi)=\sup _{j \in Z^{2 n}}\left|\mathscr{F}\left(\chi_{j} a\right)(\Xi)\right|$, 
where $\mathscr{F}$ is the Fourier transform. We have

$$
S_{0,0}^{0} \subset S_{0,0 ; 2 n+1} \subset \mathscr{A} \subset C^{0}\left(\mathrm{R}^{2 n}\right) \cap L^{\infty}\left(\mathrm{R}^{2 n}\right),
$$

where $S_{0,0}^{0}=C_{b}^{\infty}\left(\mathrm{R}^{2 n}\right)$ is the space of $C^{\infty}$ functions on $\mathrm{R}^{2 n}$ which are bounded as well as all their derivatives, $S_{0,0 ; 2 n+1}$ is the set of functions defined on $\mathrm{R}^{2 n}$ such that $\left|\left(\partial_{\xi}^{\alpha} \partial_{x}^{\beta} a\right)(x, \xi)\right| \leq C_{\alpha \beta}$ for $|\alpha|+|\beta| \leq 2 n+1$. A is a Banach algebra for the multiplication with the norm $\|a\|_{\mathscr{A}}=\left\|\omega_{a}\right\|_{L^{1}\left(\mathrm{R}^{2 n}\right)}$.

THEOREM 4.3 ([6, Theorem 2.5.10]). For all non-negative function a defined on $\mathrm{R}^{2 n}$ satisfying $a^{(4)} \in \mathscr{A}$, then the operator $a^{w}$ is semi-bounded from below. More precisely,

$$
a^{w}+C_{n}\left\|a^{(4)}\right\|_{\mathscr{A}} \geq 0
$$

where $C_{n}$ depends only on the dimension $n$.

\subsection{Proof of Theorem 4.1}

We shall use the partition of unity $\left(\varphi_{Y}\right)_{Y \in \mathrm{R}^{2 n}}$ given in Theorem 2.4. Let $\left(\psi_{Y}\right)_{Y \in \mathrm{R}^{2 n}}$ be a family of real-valued functions supported in $U_{Y, 2 r}$, equal to 1 on $U_{Y, r}$ and

$$
\sup _{Y \in \mathrm{R}^{2 n}}\left\|\psi_{Y}\right\|_{S(1, g)}^{(k)} \leq C\left(k, r, C_{0}\right)
$$

Indeed, with the same notations as in the proof of Theorem 2.4, the function $\psi_{Y}(X)=\chi_{0}\left(\frac{1}{2} r^{-2} g_{Y}(X-Y)\right)$ satisfies the requirements. Then with $a_{Y}=\varphi_{Y} a$, we write

$$
\psi_{Y} \sharp a_{Y} \sharp \psi_{Y}=a_{Y}+r_{Y} .
$$

Lemma 4.4 (Estimate for $r_{Y}$ ). For all $k, N \in \mathrm{N}$, there exist $C=C(k, N$, $\left.C_{0}\right)>0, l=l\left(k, N, C_{0}\right) \in \mathrm{N}$ such that for all $X \in \mathrm{R}^{2 n}, T \in \mathrm{R}^{2 n}$ with $g_{Y}(T) \leq 1$,

$$
\left|r_{Y}^{(k)}(X) T^{k}\right| \leq C\left\|a_{Y}\right\|_{S\left(\lambda_{g}(Y)^{2}, g_{Y}\right)}^{(l)}\left(1+g_{Y}^{\sigma}\left(X-U_{Y, 2 r}\right)\right)^{-N} .
$$

Moreover, there exist $C_{1}=C_{1}\left(n, C_{0}, N_{0}\right)>0, l_{1}=l_{1}\left(n, C_{0}, N_{0}\right) \in \mathrm{N}$ such that

$$
\left\|\int_{\mathrm{R}^{2 n}} r_{Y}^{w}\left|g_{Y}\right|^{1 / 2} d Y\right\|_{\mathscr{L}\left(L^{2}\left(\mathrm{R}^{n}\right)\right)} \leq C_{1}\|a\|_{S\left(\lambda_{g}^{2}, g\right)}^{\left(l_{1}\right)},
$$

To prove Lemma 4.4, we use the biconfinement estimate for the remainders, the proof of which can be found in [6, section 2.3]. 
Theorem 4.5 ([6, Theorem 2.3.4], biconfinement estimate). Let $g_{1}, g_{2}$ be two positive-definite quadratic forms on $\mathrm{R}^{2 n}$ with $g_{j} \leq g_{j}^{\sigma}$. Let $a_{j}, j=1,2$ be $g_{j}$-confined in $U_{j}$, a $g_{j}$-ball of radius $\leq 1$. Recall (2.20)

$$
\begin{aligned}
& r_{p}\left(a_{1}, a_{2}\right)(X) \\
& =\left(a_{1} \sharp a_{2}\right)(X)-\sum_{0 \leq k<p} \frac{1}{j !}\left(i \pi\left[D_{X_{1}}, D_{X_{2}}\right]\right)^{j}\left(a_{1}\left(X_{1}\right) a_{2}\left(X_{2}\right)\right)_{\mid X_{1}=X_{2}=X} .
\end{aligned}
$$

Then for all $k, l, p \in \mathrm{N}$, for all $X, T \in \mathrm{R}^{2 n}$, we have

$$
\begin{aligned}
&\left|\left(r_{p}\left(a_{1}, a_{2}\right)\right)^{(k)}(X) T^{k}\right| \leq A_{k, N, p}\left(g_{1}+g_{2}\right)(T)^{k / 2} \Lambda_{1,2}^{-p} \\
& \times\left(1+\left(g_{1}^{\sigma} \wedge g_{2}^{\sigma}\right)\left(X-U_{1}\right)+\left(g_{1}^{\sigma} \wedge g_{2}^{\sigma}\right)\left(X-U_{2}\right)\right)^{-N / 2}
\end{aligned}
$$

with $A_{k, N, p}=C(k, N, p, n)\left\|a_{1}\right\|_{g_{1}, U_{1}}^{(l)}\left\|a_{2}\right\|_{g_{2}, U_{2}}^{(l)}, l=2 n+1+k+p+N$ and

$$
\Lambda_{1,2}=\inf _{T \in \mathrm{R}^{2 n}, T \neq 0}\left(\frac{g_{1}^{\sigma}(T)}{g_{2}(T)}\right)^{1 / 2}=\inf _{T \in \mathrm{R}^{2 n}, T \neq 0}\left(\frac{g_{2}^{\sigma}(T)}{g_{1}(T)}\right)^{1 / 2} .
$$

Now we use Theorem 4.5 to prove Lemma 4.4.

Proof of Lemma 4.4. By the asymptotic formula (2.24), we have

$$
\psi_{Y} \sharp a_{Y}=a_{Y}+\frac{1}{4 i \pi} \underbrace{\left\{\psi_{Y}, a_{Y}\right\}}_{=0}+r_{2}\left(\psi_{Y}, a_{Y}\right),
$$

since $\psi_{Y}=1$ on the support of $a_{Y}$. The symbol $\psi_{Y}$ is $g_{Y}$-confined in $U_{Y, 2 r}$ and $a_{Y}$ is $g_{Y}$-confined in $U_{Y, r}$, and moreover, we have

$$
\begin{aligned}
\forall l \in \mathrm{N}, & \left\|\psi_{Y}\right\|_{g_{Y}, U_{Y, 2 r}}^{(l)}=\left\|\psi_{Y}\right\|_{S\left(1, g_{Y}\right)}^{(l)}, \\
& \left\|a_{Y}\right\|_{g_{Y}, U_{Y, r}}^{(l)}=\lambda_{g}(Y)^{2}\left\|a_{Y}\right\|_{S\left(\lambda_{g}(Y)^{2}, g_{Y}\right)}^{(l)} .
\end{aligned}
$$

Applying (4.6) to $r_{2}\left(\psi_{Y}, a_{Y}\right)$, we have for all $k, N \in \mathrm{N}$, there exist $C(k, N$, $n)>0, l(k, N, n) \in \mathrm{N}$ such that for all $X, T \in \mathrm{R}^{2 n}$,

(4.8) $\left|\left(r_{2}\left(\psi_{Y}, a_{Y}\right)\right)^{(k)}(X) T^{k}\right|$

$$
\begin{aligned}
& \leq C(k, N, n)\left\|\psi_{Y}\right\|_{g_{Y}, U_{Y, 2 r}}^{(l)}\left\|a_{Y}\right\|_{g_{Y}, U_{Y, r}}^{(l)} g_{Y}(T)^{k / 2} \Lambda_{1,2}^{-2}\left(1+g_{Y}^{\sigma}\left(X-U_{Y, 2 r}\right)\right)^{-N} \\
& \leq C(k, N, n)\left\|\psi_{Y}\right\|_{S\left(1, g_{Y}\right)}^{(l)}\left\|a_{Y}\right\|_{S\left(\lambda_{g}(Y)^{2}, g_{Y}\right)}^{(l)} g_{Y}(T)^{k / 2}\left(1+g_{Y}^{\sigma}\left(X-U_{Y, 2 r}\right)\right)^{-N},
\end{aligned}
$$

noticing here $\Lambda_{1,2}$ defined in (4.7) is equal to $\lambda_{g}(Y)$. An analogous estimate as (4.8) holds for $r_{2}\left(a_{Y}, \psi_{Y}\right)$. In our case, we write $r_{Y}$, which is defined in (4.3),

$$
r_{Y}=\left(\psi_{Y} \sharp a_{Y}-a_{Y}\right) \sharp \psi_{Y}+\left(a_{Y} \sharp \psi_{Y}-a_{Y}\right)=r_{2}\left(\psi_{Y}, a_{Y}\right) \sharp \psi_{Y}+r_{2}\left(a_{Y}, \psi_{Y}\right) \text {. }
$$


Then the estimate (4.4) follows from (4.8) and (3.5). Furthermore, for any $k, N \in \mathrm{N}$, there exist $C=C\left(k, N, n, C_{0}\right)>0, l=l\left(k, N, n, C_{0}\right) \in \mathrm{N}$ such that

$$
\left\|\bar{r}_{Y} \sharp r_{Z}\right\|_{S\left(1, g_{Y}+g_{Z}\right)}^{(k)} \leq C\left\|a_{Y}\right\|_{S\left(\lambda_{g}(Y)^{2}, g_{Y}\right)}^{(l)}\left\|a_{Z}\right\|_{S\left(\lambda_{g}(Z)^{2}, g_{Z}\right)}^{(l)} \delta_{2 r}(Y, Z)^{-N} .
$$

Thus we can apply Cotlar's lemma and get the estimate (4.5).

Lemma 4.6 (Estimate for $\psi_{Y}$ ). For all $k, N \in \mathrm{N}$, there exist $C=C(k, N$, $\left.C_{0}\right)>0, l=l\left(k, N, C_{0}\right) \in \mathrm{N}$ such that for all $X \in \mathrm{R}^{2 n}, T \in \mathrm{R}^{2 n}$ with $g_{Y}(T) \leq 1$,

$$
\left|\left(\psi_{Y} \sharp \psi_{Y}\right)^{(k)}(X) T^{k}\right| \leq C\left(\left\|\psi_{Y}\right\|_{S\left(1, g_{Y}\right)}^{(l)}\right)^{2}\left(1+g_{Y}^{\sigma}\left(X-U_{Y, 2 r}\right)\right)^{-N} .
$$

Moreover, there exists $C_{2}=C_{2}\left(n, C_{0}, N_{0}\right)>0$ such that

$$
\left\|\int \psi_{Y}^{w} \psi_{Y}^{w}\left|g_{Y}\right|^{1 / 2} d Y\right\|_{\mathscr{L}\left(L^{2}\left(\mathrm{R}^{n}\right)\right)} \leq C_{2} .
$$

Proof. The inequality (4.9) follows immediately from (3.5). And it follows from (3.5), (4.2) and (4.9) that for all $k, N \in \mathrm{N}$,

$$
\left\|\left(\psi_{Y} \sharp \psi_{Y}\right) \sharp\left(\psi_{Z} \sharp \psi_{Z}\right)\right\|_{S\left(1, g_{Y}+g_{Z}\right)}^{(k)} \leq C \delta_{2 r}(Y, Z)^{-N},
$$

for some $C=C\left(k, N, n, C_{0}\right)>0$. Then by choosing $N=2 N_{1}$ and using Cotlar's lemma, we get the estimate (4.10).

END OF THE PROOF OF THEOREM 4.1. The symbol $a_{Y}$ is non-negative and uniformly in $S\left(\lambda_{g}(Y)^{2}, g_{Y}\right)$, so that we can apply the Fefferman-Phong inequality (Theorem 4.3) for the constant metric $g_{Y}$ to get

$$
a_{Y}^{w}+C(n)\left\|a_{Y}\right\|_{S\left(\lambda_{g}(Y)^{2}, g_{Y}\right)}^{(l(n))} \geq 0 .
$$

By Proposition 2.5 and Lemma 2.8, we have

$$
\left\|a_{Y}\right\|_{S\left(\lambda_{g}(Y)^{2}, g_{Y}\right)}^{(l(n))} \leq C\left(n, C_{0}, N_{0}\right)\|a\|_{S\left(\lambda_{g}^{2}, g\right)}^{(l(n))},
$$

so that

$$
a_{Y}^{w}+C_{3}\|a\|_{S\left(\lambda_{g}^{2}, g\right)}^{(l(n))} \geq 0
$$


where $C_{3}=C_{3}\left(n, C_{0}, N_{0}\right)>0, l(n) \in \mathrm{N}$ are constants. Combining (4.3), (4.5), (4.10) and (4.11), we obtain

$$
\begin{aligned}
a^{w} & =\int_{\mathrm{R}^{2 n}} a_{Y}^{w}\left|g_{Y}\right|^{1 / 2} d Y \\
& =\int_{\mathrm{R}^{2 n}} \psi_{Y}^{w} a_{Y}^{w} \psi_{Y}^{w}\left|g_{Y}\right|^{1 / 2} d Y-\int_{\mathrm{R}^{2 n}} r_{Y}^{w}\left|g_{Y}\right|^{1 / 2} d Y \\
& \geq-C_{3}\|a\|_{S\left(\lambda_{g}^{2}, g\right)}^{(l(n))} \int_{\mathrm{R}^{2 n}} \psi_{Y}^{w} \psi_{Y}^{w}\left|g_{Y}\right|^{1 / 2} d Y-C_{1}\|a\|_{S\left(\lambda_{g}^{2}, g\right)}^{\left(l_{1}\right)} \\
& \geq-C\|a\|_{S\left(\lambda_{g}^{2}, g\right)}^{(l)},
\end{aligned}
$$

for some $C=C\left(n, C_{0}, N_{0}\right)>0$ and $l=l\left(n, C_{0}, N_{0}\right) \in \mathrm{N}$. The proof of Theorem 4.1 is complete.

AcKNOWLEDGEMENTS. The author would like to thank Nicolas Lerner for numerous helpful discussions and for his continual encouragement. This work is funded by a Fondation CFM-JP Aguilar grant.

\section{REFERENCES}

1. Bony, J-M., Sur l'inégalité de Fefferman-Phong, Exp. No. III (16 pages) in: Séminaire: Équations aux Dérivées Partielles, 1998-1999, École Polytech., Palaiseau 1999.

2. Bony, J-M., and Chemin, J-Y., Espaces fonctionnels associés au calcul de Weyl-Hörmander, Bull. Soc. Math. France 122 (1994), 77-118.

3. Boulkhemair, A., On the Fefferman-Phong inequality, Ann. Inst. Fourier (Grenoble) 58 (2008), 1093-1115.

4. Dencker, N., The resolution of the Nirenberg-Treves conjecture, Ann. of Math. (2) 163 (2006), 405-444.

5. Hörmander, L., The Analysis of Linear Partial Differential Operators III, Pseudo-Differential Operators, Springer, Berlin 2007.

6. Lerner, N., Metrics on the Phase Space and Non-Selfadjoint Pseudo-Differential Operators, Pseudo-Differential Operators. Theory and Appl. 3, Birkhäuser, Basel 2010.

7. Lerner, N., and Morimoto, Y., On the Fefferman-Phong inequality and a Wiener-type algebra of pseudodifferential operators, Publ. Res. Inst. Math. Sci. 43 (2007), 329-371.

INSTITUT DE MATHÉMATIQUES DE JUSSIEU

UNIVERSITÉ PIERRE-ET-MARIE-CURIE (PARIS 6)

4 PLACE JUSSIEU

75005 PARIS

FRANCE

E-mail: wendeng@math.jussieu.fr 